\title{
A new delay forecasting system for the Passenger Information Control system (PIC) of the Tokaido-Sanyo Shinkansen
}

\author{
K. Fukami, H. Yamamoto, T. Hatanaka \& T. Terada \\ Central Japan Railway Company, Japan
}

\begin{abstract}
The former delay forecasting system for the PIC calculates the delay by adding a fixed station-to-station running time to the departure time. However the system has two problems. The first problem is that it cannot reflect the delay that occurs between stations, which is very important because the distance between stations of the Tokaido-Sanyo Shinkansen is very long. The second problem is that the delay often changes until the train departs the terminal station owing to transportation changes by the conductors. To solve these problems, we have developed a new delay forecasting system for the PIC of the Tokaido-Sanyo Shinkansen that simulates actual train movement.

The system mentioned above has been used since July 2003. This system has offered precise delay information since then, and has made great contributions towards improving the service level of Tokaido-Sanyo Shinkansen.

Keywords: simulation, passenger interface, traffic control.
\end{abstract}

\section{Introduction}

The Tokaido-Sanyo Shinkansen, the main artery of the Japanese transport network, is a very punctual railway system. When delays occur, passengers expect precise delay information. To satisfy such demand, PIC reports delay of the trains to departure indicators and automatic announcement systems at stations. To improve the service level of Tokaido-Sanyo Shinkansen, we have developed a new delay forecasting system for the PIC that simulates actual train movement. 


\section{Features of the new delay forecasting system for the PIC}

\subsection{Simulation of actual train movement}

The former delay forecasting system for the PIC calculates the delay by adding fixed station-to-station running time to the departure time. However, it cannot reflect the delay that occurs between stations, which is very important because the distance between stations of the Tokaido-Sanyo Shinkansen is very long.

To solve this problem, we have developed a new delay forecasting system for the PIC that simulates actual train movement and reflects the delay between stations. Using the train tracking information, the system calculates the current position and speed of all trains that exist in Tokaido-Sanyo Shinkansen. On the basis of the information, it simulates actual train movement and generates the forecasted diagram and delay every 15 seconds.

Fig.1 shows an example of the guidance when the train stops between stations. The former delay forecasting system cannot indicate the delay even if the train delays between stations and passengers have to wait for the train on the platform. On the other hand, the new delay forecasting system can indicate the delay between stations, and passengers are able to wait in a comfortable waiting room.

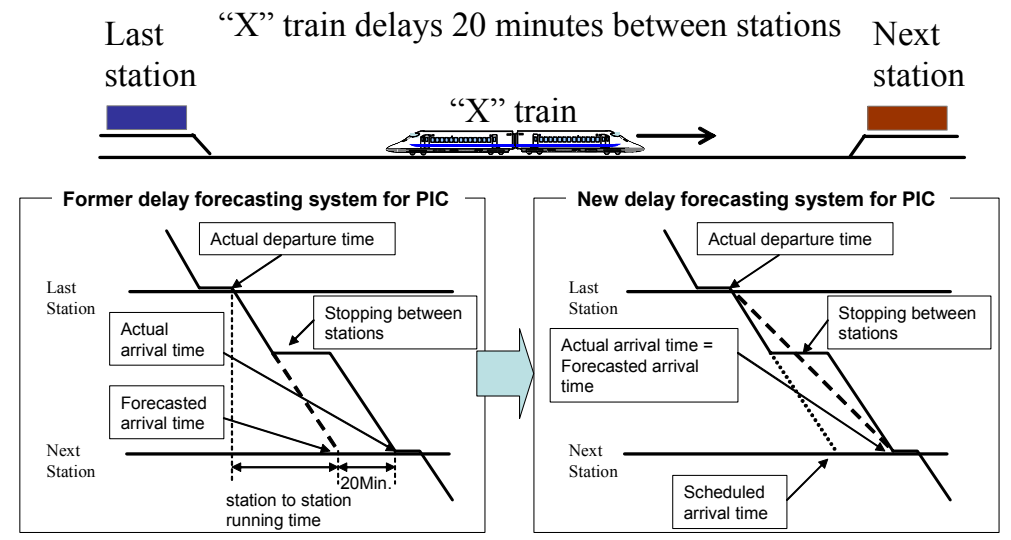

Figure 1: Example of the guidance when the train stops between stations.

\subsection{Generation of delay status information}

The former delay forecasting system calculates the delay of shuttle train using the previous operating train and transmits only the delay to the PIC. However, when the delay occurs, conductors modify diagram and car scheduling to minimize the delay. It causes a decrease in the delay of the shuttle train, and if passengers believe the delay indicated before the modification, they are likely to miss their reserved train.

To solve the problem, the new system generates the delay status information as well as the delay. Using this information, departure indicators do not indicate 
the real delay, but the delay status information such as "delay expected", until the shuttle train departs the origin station.

Fig. 2 shows the example of the shuttle train's guidance. The situation is as follows. 'Nozomi 8' delays and the shuttle train 'Nozomi 21' expects to delay 30 minutes at Shin-yokohama station. After conductors modify car scheduling from 'Nozomi 8' -> 'Nozomi 21' to 'Deadhead 9890' -> 'Nozomi 21', the delay of 'Nozomi 21' decreases to 0 minutes. In this case, the former delay forecasting system indicates that the delay of 'Nozomi 21' changes from "about 30 min" to " 0 min". If passengers who reserve 'Nozomi 21 ' believe the indication "about 30 min", they are likely to miss the train. On the other hand, the new delay forecasting system does not shows the concrete delay until the shuttle train departs the origin station. In this case, it indicates the delay that changes from "delay expected" to " 0 min", and it does not lead the passengers to miss the train.

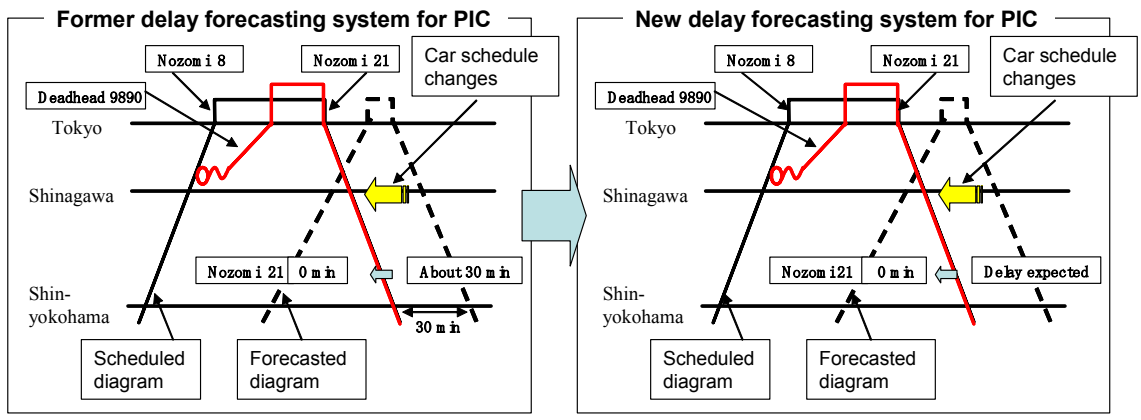

Figure 2: Example of the shuttle train's guidance.

\section{System configuration}

\subsection{System Architecture}

Fig. 3 shows the system architecture of the new delay forecasting system for the PIC. Former delay forecasting system exists in EDP (Electric Data Processing computer) system. However, the new delay forecasting system needs train tracking information which is managed in the PRC (Programmed Route Control) system. Therefore, the delay forecasting system is developed in PRC system, and to influence the existing function, we employed a new workstation called DFP (Diagram Forecasting Processor). To enhance the reliability, we adopted dual system to the DFP.

\subsection{Software Architecture}

The new diagram forecasting system consists of 3 functions, "Train tracking function", "Current condition detecting function" and "Train movement simulation function". 


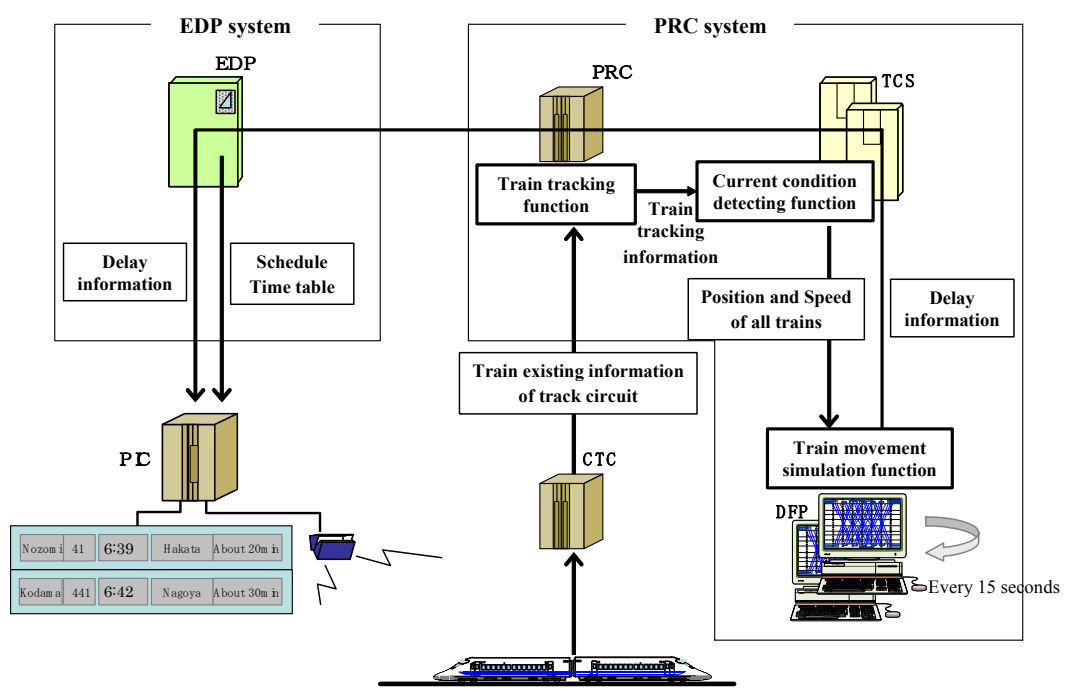

Figure 3: System configuration.

\subsubsection{Train tracking function}

Train tracking function in PRC tracks the trains using the track circuits' sequential ON/OFF movement, which is obtained from CTC (Centralized Traffic Control System). This function renews the train tracking information and sends it to the current condition detecting function every time the function receives information from CTC.

\subsubsection{Current condition detecting function}

Current condition detecting function in TCS (Traffic Communication Server) calculates the speed and the position of all trains every time the train tracking information as follows.

First, it calculates ATC (Automatic Train Control system) speed signal by the existence track of previous train. After that, the speed of all trains is calculated:

1) If the train exists in the border of a track circuit, the speed of the train is calculated as follows:

Speed $=$ the length of the train / the time between ON/OFF movement of the track circuit

2) If the train exists in the middle of a track circuit, the speed of the train is calculated as follows:

a) The target speed is calculated, using minimum of ATC speed signals, speed limit and maximum speed of the car.

b) The train's acceleration or deceleration is decided by comparing the previous speed with the target speed.

c) The speed of the train is calculated by the decision of the b) and the previous speed.

3) Finally it renews the position of all trains, using the calculated speed above, and sends it to the train movement simulation function. 


\subsubsection{Train movement simulation function}

Train movement simulation function in DFP simulates the train movement on the basis of the current speed and position, and makes the forecasted diagram and the delay of all existing trains. This function simulates the train movement reflecting all conditions such as the train performance, ATC signal speed, slope of the position, speed limits and so on. To reflect the delay between stations, it is sufficient to forecast using the train movement simulation up to the next station. In order to reduce CPU load, after this function simulates the next station's delay using the train movement simulation, it calculates the next 5 stations' delay using the conventional method, which adds station-to-station running time that reflects temporary speed limits.

This function also generates delay status information on the basis of the position of the trains and car scheduling.

Every 15 seconds, the function generates the delay and delay status information, and they are transmitted to the PIC which controls departure indicators and automatic announcement systems at stations by way of TCS, PRC and EDP.

\section{Conclusion}

The information of the PIC is very important, and the position and the speed of trains differ every time, therefore we had to test the system for more than a year. In order to improve the quality, we have also developed some debugging tools that replay the conditions, display the running profile, detect the change of the delay, etc.

The system mentioned above has been used since July 2003. Owing to sufficient tests, this system has offered precise delay to the passengers since then. It has made great contributions towards improving the service level of Tokaido-Sanyo Shinkansen.

\section{Reference}

[1] Fukami, K. \& Yamamoto, H., A new diagram forecasting system for the Tokaido-Sanyo Shinkansen. World Congress on Railway Research, Koln, Germany, No 86, 2001. 